\title{
DETERMINAÇÃO DA QUANTIDADE DE ÁGUA NO ÁLCOOL ETÍLICO HIDRATADO COMBUSTÍVEL ATRAVÉS DA TÉCNICA DA ATENUAÇÃO DA RADIAÇÃO GAMA
}

\section{DETERMINATION OF WATER IN HYDRATED ETHANOL FUEL THROUGH GAMMA RADIATION ATTENUATION TECHNIQUE}

\author{
José Martins de Oliveira Jr. ${ }^{1}$, Ítalo Castilho Oliveira Silva ${ }^{2}$, \\ Bruno Rodrigues Malaquias ${ }^{2}$ \\ ${ }^{1}$ Universidade de Sorocaba - UNISO, Campus Seminário, Caixa Postal 578, \\ Av. Dr. Eugênio Salerno, 100, Centro, 18035-430, Sorocaba, SP, Brasil. \\ 2 Colégio Dom Aguirre, Campus Seminário, Caixa Postal 578, \\ Av. Dr. Eugênio Salerno, 100, Centro, 18035-430, Sorocaba, SP, Brasil.
}

Recebido para publicação em: 30/08/2009

Aceito para publicação em: 01/12/2009

\section{RESUMO}

Neste trabalho, apresenta-se uma técnica alternativa e não convencional para a investigação da quantidade de água presente no álcool etílico hidratado usado como combustível. A técnica padrão para essa determinação é o uso de densímetros. Propõe-se, neste texto, o uso da técnica da absorção da radiação gama, ao atravessar um meio material. Mede-se o coeficiente de atenuação linear das amostras de álcool etílico hidratado e, a partir deste, calcula-se a quantidade de água, em volume, presente no álcool etílico hidratado combustível. Utiliza-se uma montagem experimental, que consiste de uma fonte emissora de raios gama de Amerício-241 ( $\mathrm{E}=59,6 \mathrm{keV}$ e atividade de 3,7x109 $\mathrm{Bq}$ ), um detector de $\mathrm{NaI}(\mathrm{Tl})$ e uma eletrônica padrão para o processamento de sinais. Foram analisadas amostras de álcool etílico hidratado combustível coletadas em estabelecimentos comerciais localizados na cidade de Sorocaba, SP, Brasil, e os resultados obtidos mostram a viabilidade da técnica.

Palavras-Chave: Atenuação de raios gama. Álcool etílico hidratado. Combustível, densidade. Quantidade de água.

\begin{abstract}
In this work, is presented an alternative and non conventional technique for evaluate the water quantity present in the hydrated ethyl alcohol used as fuel. The
\end{abstract}


standard technique used in this kind of measure is the use of a densimeter. The proposal of this work is based on the measure of the linear attenuation coefficient of gamma-rays beam by hydrated ethyl alcohol. The water quantity, in volume, in the hydrated ethyl alcohol can be determined by knows of linear attenuation coefficient. The experimental set-up, make use of an Americium-241 gamma- ray source $\left(\mathrm{E}=59,6 \mathrm{keV}\right.$ and $3,7 \times 10^{9} \mathrm{~Bq}$ of activity), a $\mathrm{NaI}(\mathrm{Tl})$ scintillation detector and a standard signal processing. In this work, some samples of hydrated ethyl alcohol, collected in some commercial establishment, located in Sorocaba city, SP, Brazil, were analyzed and the results shown the feasibility of technique.

Keywords: Gamma ray attenuation. Hydrated ethyl alcohol. Fuel, density. Water quantity.

\section{INTRODUÇÃO}

Desde a descoberta da radioatividade por $\mathrm{H}$. Becquerel, em 1896, começaram a surgir ideias de aplicações dessa nova ciência, hoje conhecida como física das radiações ionizantes. Um dos primeiros trabalhos experimentais que descreve o uso das radiações ionizantes foi desenvolvido por Belcher et al. (1950), na década de 50. Nos anos seguintes, novas aplicações para as radiações ionizantes começaram a surgir, nos mais variados campos das ciências: estudos para determinação da densidade de materiais feitos à base de cimento (SMITH; WHIFFIN, 1952), determinação da densidade de solos (VOMOCIL, 1954), medidas da concentração de partículas sólidas em fluídos (BARTOLOMEW, 1957), estudos envolvendo a concentração de metais pesados em solução aquosa (THURNAU, 1957), medidas de densidade e do conteúdo de água em madeiras (LOOS, 1961), etc. O uso do radioisótopo Amerício-241 $\left({ }^{241} \mathrm{Am}\right)$ como fonte emissora de radiação gama foi proposto por Miller (1955), em 1955, e utilizado em medidas de concentração de sais de Plutônio em solução aquosa. $\mathrm{O}^{241} \mathrm{Am}$ possui um tempo de meia vida de 458 anos e $86 \%$ dos raios gama emitidos por uma fonte de ${ }^{241} \mathrm{Am}$ possuem energia de $59,6 \mathrm{keV}$, o que torna o uso desse emissor gama muito interessante, pois raios gama de energia baixa possuem maior probabilidade de interagir com os constituintes que formam o meio em estudo. Nos dias de hoje, as radiações ionizantes provenientes das mais variadas fontes são utilizadas em ensaios físico-químicos, em todas as áreas das ciências.

Neste trabalho, sugere-se o uso da técnica de atenuação da radiação gama, proveniente de uma fonte de ${ }^{241} \mathrm{Am}$, para a determinação da porcentagem de água presente no álcool etílico hidratado, usado como combustível. Analisou-se uma série de amostras de álcool etílico hidratado, colhidas em postos que comercializam esse combustível, escolhidos aleatoriamente e situados na cidade de Sorocaba, SP, Brasil.

\section{MATERIAIS E MÉTODOS}

O método utilizado está baseado na atenuação da radiação gama, ao atravessar um meio material. No processo de interação da radiação com a matéria, fótons podem ser completamente absorvidos do feixe incidente ou espalhados em alguma direção. $\mathrm{O}$ processo de remoção dos fótons (espalhados ou absorvidos) segue uma lei exponencial, sendo que o número de fótons removido do feixe incidente, por uma camada de material absorvedor com uma determinada espessura, é proporcional a essa espessura e ao número de fótons incidente. Tal fato é descrito pela lei de atenuação de Lambert-Beer (SIEGBAHN, 1979). Considere um feixe de raios gama monoenergético incidindo sobre um material de espessura $x \mathrm{e}$ densidade $\rho$, conforme mostra a Figura 1. Parte da radiação incidente é espalhada, parte é absorvida, e uma fração atravessa o material sem interagir. A intensidade $I$ (cps) do feixe emergente está relacionada à intensidade $I_{0}(\mathrm{cps})$ do feixe incidente pela lei de Lambert-Beer. Ou seja:

$$
I=I_{0} e^{-\mu^{*} \rho x}
$$


onde $x(\mathrm{~cm})$ representa a espessura total do material absorvedor, $\rho\left(\mathrm{g} / \mathrm{cm}^{3}\right)$ é a densidade do material absorvedor e $\mu^{*}\left(\mathrm{~cm}^{2} / \mathrm{g}\right)$ recebe o nome de coeficiente de atenuação de massa. As intensidades são dadas em quantidade de fótons por unidade de tempo. $\mathrm{O}$ coeficiente de atenuação de massa $\mu^{*}\left(\mathrm{~cm}^{2} / \mathrm{g}\right)$ está relacionado com o coeficiente de atenuação linear $\mu\left(\mathrm{cm}^{-1}\right)$, através da relação $\mu=\mu^{*} \rho$.

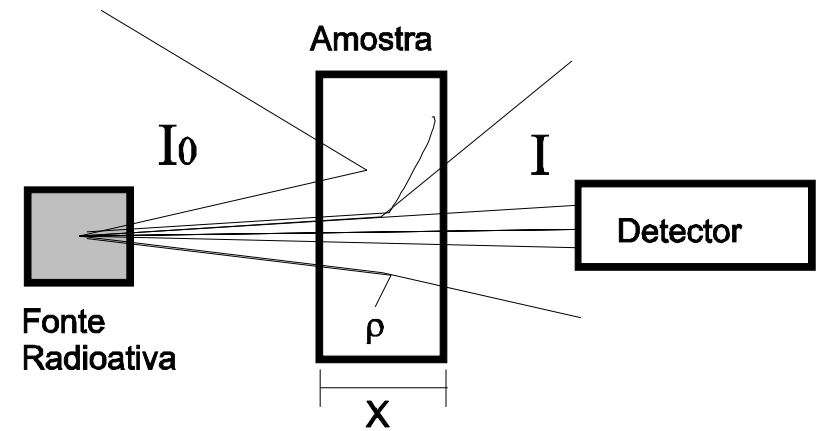

Figura 1 - Esquema mostrando a atenuação de um feixe de raios gama incidente em uma amostra de densidade $\rho$ e espessura $x$.

Ferraz e Mansell (1979) afirmaram que o coeficiente de atenuação de massa para um material heterogêneo está diretamente relacionado à composição química do material. Segundo Ferraz, o coeficiente de atenuação de massa teórico pode ser assumido como sendo o somatório dos produtos dos coeficientes de atenuação de massa de cada elemento químico que compõe o material, multiplicado pela sua respectiva porcentagem na amostra. Ou seja:

$$
\mu^{*}=\sum_{i=1}^{n}\left(R_{i} \mu_{i}^{*}\right)=R_{1} \mu_{1}^{*}+R_{2} \mu_{2}^{*}+\ldots \ldots \ldots+R_{n} \mu_{n}^{*},
$$

onde $n$ é o número que identifica o elemento químico que compõe a amostra, $R_{i}$ é a percentagem do iésimo elemento químico presente na amostra e $\mu^{*}{ }_{i}$ é o coeficiente de atenuação de massa do iésimo elemento. Considerando que as amostras de álcool combustível hidratado são constituídas de álcool e água, conforme mostrado esquematicamente na Figura 2, e que a proposta de Ferraz se aplica para essa mistura de duas substâncias, pode-se escrever:

$$
\mu^{*}=\mu_{\text {alc. }}^{*} R_{\text {allc. }}+\mu_{\text {ag. }}^{*} R_{\text {ag. } .},
$$

sendo $\mu_{\text {alc. }}^{*}$ e $\mu^{*}{ }_{\text {ag. }}$ os coeficientes de atenuação de massa do álcool e da água, respectivamente, e $R_{a b l c .}$ e $R_{a g .}$ as razões entre os volumes de álcool e de água e o volume total, respectivamente. As quantidades $R_{a l c .}$ e $R_{a g}$ foram definidas como:

$$
R_{\text {allc. }}=\frac{V_{\text {allc. }}}{V_{t}}=\frac{X_{a l c .}}{X_{t}} \quad e \quad R_{a g .}=\frac{V_{a g .}}{V_{t}}=\frac{X_{a g .}}{X_{t}},
$$

sendo $V_{a l c .}$ e $V_{a g}$. os volumes de álcool e água contidos na amostra, respectivamente, $X_{a l c}$. e $X_{a g .}$ as espessuras equivalentes de álcool e água presentes na amostra, conforme mostra a Figura 2, e $V_{t}=V_{a l c .}+V_{a g}$. e $X_{t}=X_{a l c} .+X_{a g .}$ o volume e a espessura total da amostra.

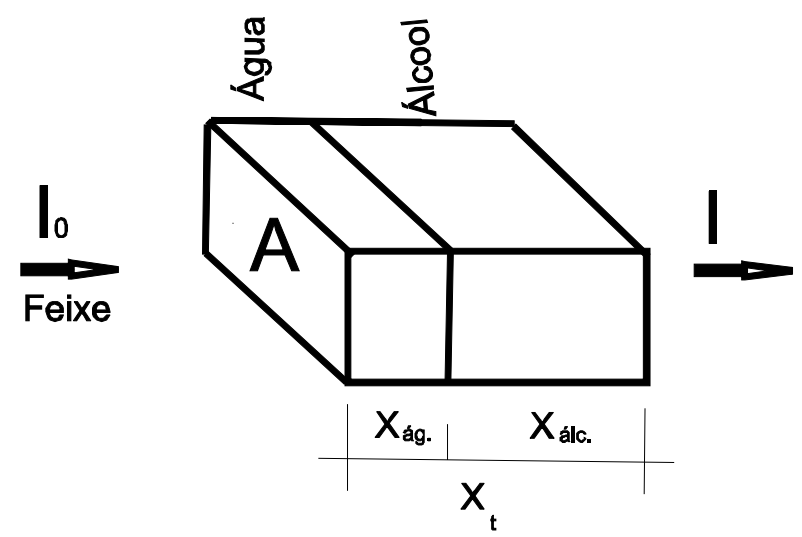

Figura 2 - Representação esquemática de uma amostra de álcool hidratado combustível, sendo $A$ a área da amostra, $X_{a g .}$ e $X_{a l c .}$ as espessuras equivalentes de água e álcool contidas na amostra, respectivamente, e $X_{t}$ a espessura total da amostra.

Substituindo as definições apresentadas na equação (4) e na equação (3), encontramos:

$\mu^{*}=\frac{\mu_{a l c .}^{*} X_{a l c .}}{X_{t}}+\frac{\mu_{a g .}^{*} X_{a g .}}{X_{t}}$, 
Usando-se a relação entre os coeficientes de atenuação de massa $\mu^{*}$ e os coeficientes de atenuação linear $\mu\left(\mu^{*}=\mu / \rho\right)$, pode-se escrever a equação (5) como:

$$
\frac{X_{t} \mu}{\rho}=\frac{\mu_{\text {alc. }} X_{\text {alc. }}}{\rho_{\text {alc. }}}+\frac{\mu_{\text {ag. }} X_{\text {ag. }}}{\rho_{\text {ag. }}}
$$

sendo $\rho_{\text {alc. }}, \rho_{\text {ag. }}$ e $\rho$ as densidades do álcool, da água e da amostra, respectivamente, e $\mu_{a l c}$, $\mu_{a g}$. $e \mu$ os coeficientes de atenuação linear do álcool da água e da amostra, respectivamente. Definindo a densidade $\rho$ da amostra, por meio de uma média ponderada, e usando como peso na média o volume de cada substância, pode-se escrever para $\rho$ :

$\rho=\frac{\rho_{\text {alc. }} V_{a l c .}+\rho_{\text {ag. }} V_{\text {ag. }}}{V_{t}}$,

Substituindo a equação (7) e as relações $R_{a g .}=X_{a g .} / X_{t}$ e $1-R_{a g .}=X_{a l c .} / X_{t}$, na equação (6) obtém-se a equação (8), que fornece a função teórica entre a quantidade de água presente no álcool hidratado combustível e o coeficiente de atenuação linear, isto é: e de um sistema padrão de processamento de sinais. Foram utilizados colimadores circulares de Chumbo, com orifício de $5 \mathrm{~mm}$ de diâmetro na saída da fonte de raios gama e com $2 \mathrm{~mm}$ de diâmetro na frente do detector. O sistema foi calibrado usando-se uma amostra de água e uma de álcool etílico $\left(\mathrm{CH}_{3} \mathrm{CH}_{2} \mathrm{OH}\right)$ de alta pureza (99.5\% de álcool) que continha no máximo $0.2 \%$ de água, segundo o fabricante (marca ECIBRA ${ }^{\circledR}$, Ind. e Com. de Produtos Químicos Ltda., Lote $\mathrm{n}^{0}: 17545$, fabricado em maio de 2008). Foram feitas medidas para se obter o coeficiente de atenuação linear do álcool etílico e da água. Mediu-se, ainda, o coeficiente de atenuação linear do álcool etílico diluído em água, em diferentes concentrações. Esse coeficiente também foi determinado teoricamente usando-se o programa Xcom (HUBBEL; BERGER, 1987).

Os resultados obtidos foram utilizados para testar o modelo teórico representado pela equação (8) e na construção da curva de calibração da proporção de água $\left(R_{a g}\right.$. ) versus coeficiente de atenuação linear. Essa curva, por sua vez, foi utilizada na determinação da quantidade de água contida nas amostras analisadas. $\mathrm{O}$ valor da quantidade de água obtida pelo método da atenuação da radiação gama também foi comparado com a metodologia tradicional, ou seja, via medida da densidade.

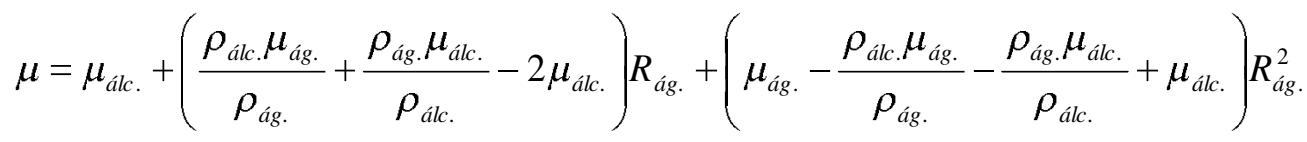

A equação (8) mostra uma dependência quadrática entre a quantidade de água $R_{\text {ag. }}$ e o coeficiente de atenuação linear $\mu$. Conhecendo-se os coeficientes de atenuação linear da água e do álcool etílico, e suas respectivas densidades, pode-se determinar a quantidade de água $R_{a g .}$ presente na amostra, desde que se conheça o coeficiente de atenuação linear da amostra.

A montagem experimental utilizada na determinação dos coeficientes de atenuação consiste de um sistema para espectrometria gama, sendo composto por uma fonte emissora de raios gama de Amerício-241 $\left({ }^{241} \mathrm{Am}\right)$ com atividade de $3.7 \times 10^{9} \mathrm{~Bq}$ e que emite fótons preferencialmente na energia de 59,6 keV, de um detector de $\mathrm{NaI}(\mathrm{Tl})$, de colimadores

\section{RESULTADOS E DISCUSSÃO}

Usando-se o programa Xcom, determinaram-se teoricamente os coeficientes de atenuação de massa $\mu^{*}$, da água e do álcool etílico, e após a multiplicação desses coeficientes pelas respectivas densidades dessas substâncias $\left(\rho_{\text {agua }}=1 \mathrm{~g} / \mathrm{cm}^{3}\right.$ e $\rho_{\text {alcool }}=0.7876$ $\mathrm{g} / \mathrm{cm}^{3}$ ), obtiveram-se os coeficientes de atenuação linear teóricos para a água $\left(0.2070 \mathrm{~cm}^{-1}\right)$ e para o álcool $\left(0.1583 \mathrm{~cm}^{-1}\right)$. Os coeficientes de atenuação linear também foram obtidos experimentalmente, por meio da equação (1), sendo essas medidas feitas com álcool etílico puro, diluído em água em diferentes proporções e água. Os valores experimentais obtidos para o coeficiente de atenuação linear da água e 
do álcool foram $\mu_{a g}=(0.2007 \pm 0.0006) \mathrm{cm}^{-1}$ e $\mu_{\text {allc. }}=$ $(0.1561 \pm 0.0005) \mathrm{cm}^{-1}$, respectivamente.

Substituindo os valores teóricos das densidades e dos coeficientes de atenuação linear da água e do álcool etílico na equação (8), obtém-se a relação teórica entre quantidade de água e coeficiente de atenuação linear, isto é:

$$
\mu=0.15830+0.04742 R_{\text {ag. }}-0.00128 R_{\text {ag. }}^{2}
$$

A Figura 3 mostra os valores experimentais dos coeficientes de atenuação linear, em função da quantidade de água presente nas amostras utilizadas na calibração do sistema. Para cada amostra analisada, o volume de água usado na diluição $\left(R_{a g}\right)$, em relação ao volume total da amostra, era conhecido. A linha sólida representa o gráfico da equação (9), a linha pontilhada é o resultado que se obtém quando se ajusta aos dados experimentais uma função quadrática.

Analisando a Figura 3, observa-se que a dependência de $\mu \operatorname{com} R_{a g}$ é praticamente linear. A equação (9), por outro lado, mostra uma dependência quadrática entre $\mu$ e $R_{\text {ag.: }}$ no entanto, pode-se dizer que a representação gráfica da equação (9), mostrada pela linha sólida na Figura (3), pelo menos visualmente, é uma reta. Isso ocorre porque o coeficiente que acompanha o termo quadrático na equação (9) é pequeno quando comparado ao coeficiente do termo linear, sendo, portanto, desprezível. O melhor ajuste quadrático aos dados experimentais é dado pela equação (linha pontilhada na Figura 3) $\mu=0.015763+0.05944 R_{\text {ag. }},-$ $-0.01345 \mathrm{R}_{\text {ag. }}^{2}$, com coeficiente de correlação igual a $\mathrm{r}^{2}=0.9972$.

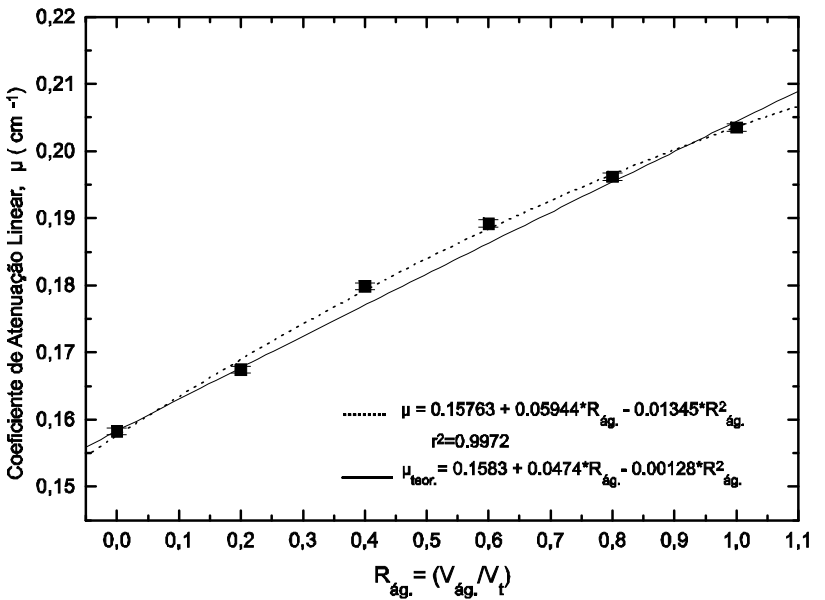

Figura 3 - Gráfico mostrando a dependência do coeficiente de atenuação linear em função da quantidade de água. A linha sólida é o gráfico da equação teórica, enquanto a linha pontilhada representa a curva quadrática que melhor se ajusta aos dados experimentais.

Desprezar o termo quadrático na equação (9) equivale a considerar que a proposta de Ferraz e Mansell representada pela equação (2) pode ser modificada, trocando-se o coeficiente de atenuação de massa pelo coeficiente de atenuação de linear. $\mathrm{O}$ resultado que se obtém para a relação entre $\mu$ e $R_{\text {ag., }}$ ao trocar-se o $\mu^{*}$ de massa pelo $\mu$ linear na equação (2), e usando as mesmas considerações feitas na dedução da equação (9), é mostrado na equação (10).

$\mu=\mu_{\text {alc. }}+\left(\mu_{\text {ag. }}-\mu_{\text {alc. }}\right) R_{\text {ag }}$

Substituindo-se os valores teóricos dos coeficientes de atenuação linear na equação (10), obtém-se a equação (11).

$$
\mu=0.1583+0.0487 R_{\text {ag }}
$$

Comparando-se as equações (8) e (10), verifica-se que a equação (8) converge para a equação (10), à medida que a densidade das substâncias se aproxima. Se as densidades forem iguais, a equação (8) torna-se a equação (10). No estudo conduzido neste trabalho, como a densidade das substâncias (álcool e água) difere por apenas $21 \%$, e a porcentagem de água no álcool etílico não deve ser grande, pois 
se está trabalhando com valores pequenos para $R_{a g}$, poder-se-ia, com certeza, utilizar a equação (10) para descrever a relação entre $\mu$ e $R_{a g .,}$ que o erro cometido seria desprezível. A Figura 4 mostra os gráficos das equações teóricas (9) (linha sólida) e (11) (linha pontilhada), nos quais se observa que até por volta de $20 \%$ de água, as curvas são coincidentes.

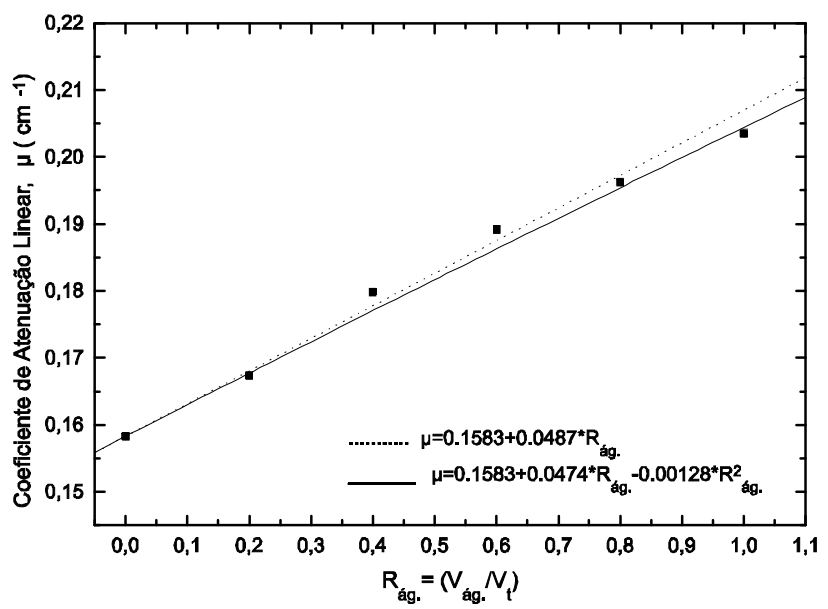

Figura 4 - Gráfico mostrando a dependência do coeficiente de atenuação linear com a quantidade de água. A linha sólida é o gráfico da equação (9), enquanto a linha pontilhada mostra o gráfico da equação (11).

Em resumo, partindo da equação (2) proposta por Ferraz e Mansell obtém-se a dependência correta

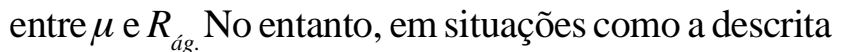
neste trabalho, poderia ser utilizada a equação (11), que é mais simples e fornece valores para a quantidade de água iguais aos obtidos via equação (9).

A Tabela 1 mostra os valores obtidos para o coeficiente de atenuação linear de amostras de álcool combustível comercializado na cidade de Sorocaba, SP, Brasil. A escolha dos postos de combustível onde as amostras seriam colhidas foi aleatória e não se informou o destino que as mesmas teriam. Alguns dos postos escolhidos possuem vinculo com uma única distribuidora (com bandeira) e outros não possuem vinculo com determinada distribuidora de combustível (sem bandeira). Esses postos serão identificados por números, seguidos pelas letras $\mathrm{CB}$ para os que trabalham com bandeira e pelas letras SB para os sem bandeira. Os resultados para a quantidade de água presente nas amostras de álcool etílico hidratado, mostrados na Tabela 1, foram obtidos usando-se: i) a relação teórica entre $\mu$ e $R_{a g}$, apresentada na equação (11); e ii) por meio da equação experimental $\left(\mu=0.015763+0.05944 R_{a g .},-0.01345 R_{a g .}^{2}\right)$ que melhor se ajusta aos dados usados na calibração.

No Regulamento Técnico $\mathrm{n}^{\circ}$ 7/2005 (2005), a Agencia Nacional de Petróleo, Gás Natural e Biocombustíveis (ANP) estabelece que, em volume, os limites de água permitidos para o álcool etílico hidratado combustível devem ficar entre $6,2 \%$ a $7,4 \%$.

Tabela 1 - Valores de coeficiente de atenuação linear $\mu \mathrm{e}$ quantidade de água $R$, de amostras de álcool combustível hidratado, coletadas em postos que comercializam esse combustível, situados na cidade de Sorocaba, SP, Brasil.

\begin{tabular}{c|c|c|c}
\hline $\begin{array}{c}\text { Posto de } \\
\text { Combustível }\end{array}$ & $\mu\left(\mathbf{c m}^{-1}\right)$ & $\mathbf{R}^{\text {Teor. }}{ }_{\text {ag. }}(\%)$ & $\mathbf{R}^{\text {Exp. }}{ }_{\text {ag. }}(\%)$ \\
\hline 1-SB & $0.1620 \pm 0.0005$ & $7.82 \pm 0.12$ & $7.48 \pm 0.15$ \\
\hline 2-CB & $0.1623 \pm 0.0005$ & $8.53 \pm 0.13$ & $8.06 \pm 0.16$ \\
\hline 3-SB & $0.1618 \pm 0.0005$ & $7.41 \pm 0.11$ & $7.14 \pm 0.14$ \\
\hline 4-CB & $0.1610 \pm 0.0005$ & $5.89 \pm 0.09$ & $5.89 \pm 0.11$ \\
\hline 5-CB & $0.1614 \pm 0.0005$ & $6.69 \pm 0.10$ & $6.55 \pm 0.13$ \\
\hline 6-SB & $0.1643 \pm 0.0005$ & $12.89 \pm 0.19$ & $11.69 \pm 0.23$ \\
\hline 7-SB & $0.1646 \pm 0.0005$ & $13.36 \pm 0.20$ & $12.07 \pm 0.24$ \\
\hline
\end{tabular}

A Figura 5 mostra os valores de $R^{\text {Teor. }}{ }_{a g .}(\%)$ e $R^{\text {Exp. }}{ }_{\text {ag. }}(\%)$, junto com os limites impostos pela ANP, para as diferentes amostras de álcool etílico hidratado analisadas. Uma análise rigorosa da Figura 5 mostra que apenas os postos de $n^{\circ} 3$ e 5 estariam dentro dos limites estabelecidos pela ANP. No entanto, os postos de $n^{\circ} 1,2$ e 4 estão muito próximos desse limite, e praticamente atendem as especificações da ANP; já as amostras coletadas nos postos de $n^{\circ} 6$ e 7 apresentaram quantidades de água muito superiores aos limites estabelecidos pela ANP, ou seja, quase o dobro do permitido. Com certeza, o álcool etílico combustível comercializado pelos estabelecimentos de $\mathrm{n}^{\circ} 6$ e 7 foi adulterado, adicionando-se mais água que o permitido.

É importante ressaltar que se está considerando, nesta discussão, que as diferenças nos valores dos coeficientes de atenuação linear provêm exclusivamente da presença de água no álcool, pois essa é a forma de falsificação mais simples, barata e utilizada por estabelecimentos que adulteram o combustível álcool hidratado. Os postos de $\mathrm{n}^{\circ} 6$ e 7 pertencem ao 


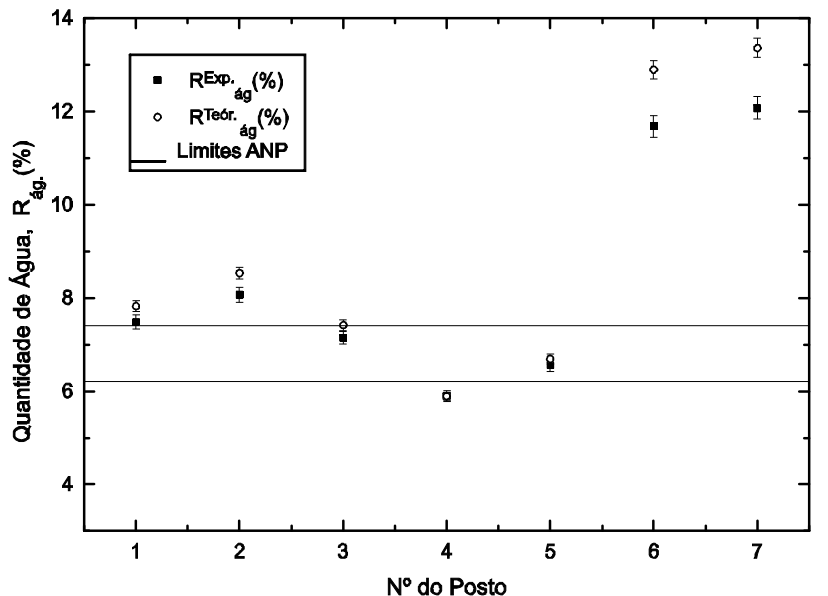

grupo dos sem bandeira.

Figura 5 - Valores da quantidade de água, em porcentagem, presentes em amostras de álcool etílico hidratado, coletadas em diferentes estabelecimentos comerciais, situados na cidade de Sorocaba, SP, Brasil. As linhas sólidas horizontais indicam os limites estabelecidos pela Agência Nacional de Petróleo e Gás Natural (ANP) para a quantidade de água.

Calculou-se, também, pela metodologia tradicional, ou seja, medindo-se a densidade das amostras, o valor da quantidade de água presente nas amostras de álcool etílico. A Figura 6 mostra os resultados desse cálculo, junto com os obtidos via método da atenuação de raios gama. O método da atenuação de raios gama fornece valores maiores para a quantidade de água e mais próximos dos valores estabelecidos pela ANP. O método da densidade indica que as amostras possuíam quantidades de água inferiores aos valores especificados pela ANP, com exceção das amostras de $n^{\circ} 6$ e 7 , que continuam apresentando valores para a quantidade de água muito superiores aos estabelecidos pela ANP. Apesar de ambos os métodos terem desvios experimentais equivalentes, acredita-se que o método da atenuação possui uma vantagem em relação ao da densidade, pois as medidas não dependem da acuidade do experimentador para a obtenção do volume de cada amostra e a precisão no método da radiação pode ser melhorada aumentando-se o tempo gasto na irradiação de cada amostra.

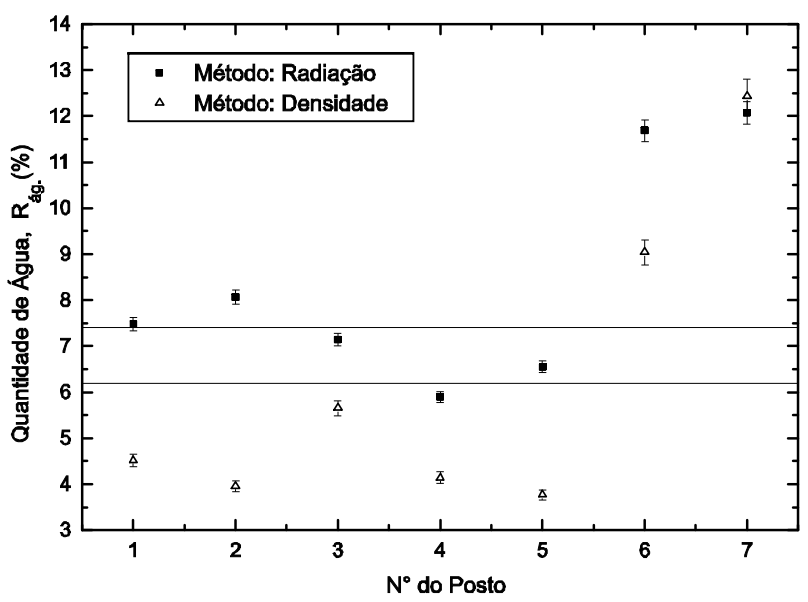

Figura 6 - Valores da quantidade de água, em porcentagem, para as amostras de álcool etílico hidratado, coletadas em diferentes estabelecimentos comerciais situados na cidade de Sorocaba, SP, Brasil. As linhas sólidas horizontais indicam os limites estabelecidos pela Agência Nacional de Petróleo e Gás Natural (ANP) para a quantidade de água. Os dados representados pelos quadrados são os valores obtidos via metodologia da atenuação de raios gama e os representados por triângulos abertos provêm da metodologia tradicional.

\section{CONCLUSÕES}

Foi analisada a quantidade de água presente em amostras de álcool etílico hidratado usado como combustível, as quais foram coletadas em estabelecimentos comerciais situados na cidade de Sorocaba, SP, Brasil. Nessa análise usou-se uma metodologia não convencional, ou seja, a técnica da atenuação da radiação gama, e a metodologia tradicional, que se baseia na medida da densidade. Fez-se uma discussão aprofundada da dependência entre o coeficiente de atenuação e a quantidade de água presente nas amostras. Mostrou-se que para o tipo de substância analisada (água e álcool), pode-se descrever a relação entre a porcentagem de água e o coeficiente de atenuação, através de uma relação linear no lugar da quadrática. Após a construção da curva de calibração do coeficiente de atenuação linear em função da quantidade de água, mostrou-se a viabilidade da 
metodologia proposta, medindo-se a porcentagem de água de algumas amostras de álcool etílico hidratado. Dados para a quantidade de água também foram obtidos via medida da densidade. As duas metodologias apresentam valores diferentes para a quantidade de água, mas não totalmente discrepantes, isto é, mostram o mesmo comportamento geral.

Os dados obtidos por meio da metodologia da atenuação de raios gama apresentaram valores para a quantidade de água mais próximos aos limites estabelecidos pela ANP do que os obtidos pelo método da densidade. Para duas das amostras analisadas $\left(n^{\circ} 6\right.$ e 7), a quantidade de água presente, obtida por ambas as técnicas, ficou muito acima dos limites estabelecidos pela ANP, enquanto a quantidade de água das outras amostras estava dentro ou muito próxima da especificação da ANP. As amostras que continham mais água vieram de postos sem bandeira. Os dados apresentados neste trabalho mostram que aproximadamente $30 \%$ dos postos pesquisados, que comercializam álcool etílico hidratado na cidade de Sorocaba, vendem esse combustível adulterado, ou seja, com maior volume de água que o permitido pela ANP, e que $100 \%$ desses postos que adulteram o combustível são postos que trabalham sem bandeira. A técnica proposta neste trabalho para se analisar a quantidade de água pode ser estendida e aplicada na análise de misturas de outros materiais.

\section{REFERÊNCIAS}

BARTOLOMEW, R. N.; CASAGRANDE, R. M. Measuring solids concentration in fluid systems by gamma-ray absorption. Ind. Eng. Chem. v.49, n.30, p.428-431, 1957.

BELCHER, D. J., CUYKENDALL, T. R.; SACK, H. S. The measurement of soil moisture and density by neutron and gamma ray scattering. Technical Development Report no. 127, Civil Aeronautics Administration, Washington D.C., 1950.

BRASIL. Agência Nacional do Petróleo e Gás Natural. Regulamento técnico ANP n.7/2005. Disponível em: <http://nxt. anp.gov.br/ NXT/ gateway.dll/leg/resolucoes_anp/2005/dezembro/ranp\%2032\%202005.xml?f=templates $\$ \mathrm{fn}=$ default. htm\&sync=1\&vid=anp:10.1048/enu $>$. Acesso em: 28 ago. 2009.

FERRAZ, E. S. B.; MANSELL, R. S. Determning water content and bulk density of soil by gamma ray attenuation methods. Agricultural Experiment Stations Institute of Food and Agricultural Sciences University of Florida, Gainesville,
Bulletin 807 (Technical), 1979.

HUBBEL, J. H.; BERGER, M. J., X-COM: photon cross section on a personal computer. NBSIR 87-3597, US Department of Commerce, National Bureau of Standards, Gaithersburg, MD, 1987.

LOOS, W. E. Gamma ray absorption and wood moisture content and density. Forest Prod. J. v.11, n.3, p.121-126, 1961.

MILLER, D. G. Americium 241 as a photon source for the gamma absortiometric technique. Oak Ridge, United States Atomic Energy Commission. Report HW 39971, 1955.

SIEGBAHN, K. Alpha, beta and gamma-ray spectroscopy. Amsterdam; New York: Oxford North-Holland Publishing Company, 1979. p.37-76.

SMITH, E. E.; A. C. WHIFFIN. Density measurement of concrete slabs using gamma radiation. Engineers, v.194, p.278-281, 1952.

THURNAU, D. H. Gamma absorptiometer for solution of heavy metal salts. Anal. Chem. v.29, n.12, p.1772-1783, 1957.

VOMOCIL, J. A. In situ measurement of bulk density of soil by gamma absorption technique. Soil Sci., v.77, p.341342, 1954. 